\section{Treating Autism Spectrum Disorder with Gluten-free and Casein-free Diet: The underly- ing Microbiota-Gut-Brain Axis Mechanisms}

Anna Cieślińska ${ }^{1}$, Elżbieta Kostyra ${ }^{1}$ and Huub FJ Savelkoul ${ }^{*}$

${ }^{1}$ Department of Biology and Biotechnology, University of Warmia and Mazury, Olsztyn, Poland

${ }^{2}$ Department of Cell Biology and Immunology group, Wageningen University \& Research, Wageningen, The Netherlands

\section{Abbreviations}

ASD - Autism Spectrum Disorder

GI - Gastrointestinal

HPA - Hypothalamic-Pituitary-Adrenal

\section{Introduction}

A growing number of children are diagnosed with Autism Spectrum Disorder (ASD), a category of neuro developmental disorders such as Autism, Pervasive Developmental Disorder-Not Otherwise Specified (PDD-NOS) and Asperger's disorder, collectively included in the DSM-V criteria. The American Psychiatric Association's Diagnostic and Statistical Manual, Fifth Edition (DSM-5) provides standardized criteria to help diagnose ASD [1]. The prevalence of ASD is at least 1 in 160 children worldwide [2-4].

It is called a spectrum disorder because patients show a varying degree and severity of symptoms [1]. The most typical symptoms are deficits in social communication and interaction, along with restricted interests, repetitive behaviors and a difficulty with imagination. Often, people with ASD avoid making eye contact with others, seem unresponsive to environmental cues and have difficulty to judge other people's emotions [5-7]. Reduced pain sensitivity is often described as well [8]. The parents can often recognize these symptoms already at one year of age. Until today, the exact etiology of ASD is unknown.

The development of ASD is complicated by the phenotypic heterogeneity of the disease and comorbidities (e.g., anxiety, hyperactivity). Genetic factors are implicated in ASD but these might account for only $10-20 \%$ of cases. Supposedly, more than 400 genes are associated with the development of ASD [6,9]. Twin studies showed a concordance rate in monozygotic twins of less than $100 \%(77 \%$ for male and $50 \%$ for female pairs), suggesting an important contribution of environmental factors in ASD development $[7,8]$. Prenatal and perinatal environmental exposures (e.g., dietary factors, maternal diabetes, stress, medications, or infections and certainly those that require antibiotic use) are associated with an increased risk of ASD $[2,10]$.

Food allergies and intolerances, besides asthma, and skin disorders such as atopic dermatitis and eczema, are co-morbidities associated with ASD despite the fact that levels of IgE antibodies, are generally not consistently different between ASD and normally developing controls [11]. Several dysregulated immune responses occur in ASD such as increased natural killer cell activity, the presence of autoantibodies directed against brain proteins, and altered cytokine profiles, leading a.o. to increased rate of middle ear infections at young age $[2,12,13]$. In addition, there is an increased prevalence of immune-mediated disorders in family members of children with ASD, including rhinitis, food allergy, and psoriasis and possibly even inflammatory bowel disease $[10,14,15]$.

\section{GFCF Diet in ASD}

Whilst diets are often followed with the purpose of losing weight, they are also used as a therapy for diseases, including Autism Spectrum Disorder (ASD) [6,16]. The Gluten-Free and Casein-Free 
(GFCF) diet is strongly promoted while there are additional diets without salicylates and phenols, and anti-candida diets since many ASD patients are affected by this yeast [17]. However, so far it has been difficult to prove the effectiveness of this diet in all patients $[10,18]$. Limited information is available on the underlying mechanism as well as explanatory factors of differences in response to the GFCF diet. Here, we focus on the mechanism of action underlying this dietary intervention as well as the origin of differences in response to the GFCF diet.

\section{Gluten-Free and Casein-Free Diet Might Alleviate Autism Spectrum Disorder Symptoms}

People with ASD are often in need of intensive care and guidance, leading to loss of parental productivity and high emotional and economic costs $[8,19]$. Therefore it is important to investigate potential treatments that alleviate the symptoms or preferably cure the disease. So far, sometimes life long, educational and behavioral interventions are used, although these therapies have limited efficacy for ASD $[6,20]$. Currently, there is growing interest in nutritional interventions for this disorder and there are promising mechanisms underlying the putative success of such interventions [19].

In 1983 it was suggested that gluten and casein containing products could cause autism-like symptoms by altering the functioning of the brain. It was proposed that gluten and casein were broken down in the intestine into small peptides that could cross the intestinal membrane and subsequently also the Blood Brain Barrier (BBB). These opioid-like peptides, called gliadorphins and casomorphins, might have a negative pharmacological effect on attention, brain maturation, social interaction and learning and thus it was hypothesized that appropriate diets would facilitate learning, social behavior, cognitive functioning and communicative skills in ASD individuals [20-23]. Based on this the orygluten-and casein-free (GFCF) dietary interventions have become of interest as a method to alleviate ASD symptoms $[6,16]$. At the moment, approximately $26 \%$ of the children with ASD follow the GFCF diet [14]. In addition to the GFCF diet, there are dietary interventions that interact with gut bacteria such as ketogenic diets, the intake of probiotics and diets high in omega-3 fatty acids $[8,19]$. Here, the focus will be on the GFCF dietary intervention only.

\section{Gut Microbiota Dysbiosis in ASD}

The importance of the relationship between the gut and the brain in autism is demonstrated by Gastrointestinal (GI) symptoms that often occur. In $23-70 \%$ of all patients symptoms such as constipation, diarrhea, flatulence and in more severe cases inflammatory bowel disease occur [7]. Also, the severity of GI symptoms is associated with the severity of ASD [24,25]. Additionally, an imbalance in gut microbiota composition is often seen in patients with ASD. Probably, this imbalance is associated with GI symptoms [19,26-29]. Dietary interventions are known to be able to restore the gut microbiota composition and toxin production by which they can positively affect the symptoms of ASD [19,27,30,31], although still is still debated $[32,33]$.

\section{The Microbiome in ASD}

The microbiome consists of over a hundred trillion bacteria collectively acting as an extension of the host's digestive, immune, metabolic and neural system [26]. The vast majority of these bacteria colonizes the GI tract during the early postnatal period and resides there throughout adult life, the commensal microbiota. The other part of the microbiome consists of transient bacteria, such as probiotics, acquired through the food $[19,34,35]$. Together they form a dynamic entity of more than a thousand different species that enrich the human genome with over 3 million genes [30].

One of the main functions of the microbiome is helping the host with metabolizing non-digestible carbohydrates, including inulin and cellulose [19]. Products of this fermentation process are metabolites such as Short-Chain Fatty Acids (SCFAs). The three SCFAs produced by the intestinal bacteria are acetic acid, propionic acid and butyric acid. SCFAs are important for the health and regulation of the intestinal membrane integrity and the development of innate and adaptive immune responses $[2,6,12,18,19]$. The gut microbiome is mainly shaped during the first months of life by different factors, including vaginal delivery, the host's genome, breastfeeding or formula feeding, use of antibiotics, GI infections and stress [2,19]. Diet is a factor that influences the bacterial composition also later in life $[8,24,36,37]$.

\section{Microbiota Dysbiosis in ASD}

Compelling evidence points towards abnormalities in gut microbiota composition in children with ASD, but it still debated whether these abnormalities contribute to development of ASD symptoms or that the diet and/or medication of the child leads to imbalances in the gut microbiota. Changes in relative distribution of microbiota (dysbiosis) were reported in ASD children with increased relative abundance of Bacteroidetes, Proteobacteria, and Verrucomicrobia, but with lower abundance of Firmicutes and Actinobacteria [17,19].

At the genus level, significant reductions were found between children with ASD compared with non affected controls in the relative abundance of Prevotella, Coprococcus, Enterococcus, Lactobacillus, Streptococcus, Lactococcus, Staphylococcu, Ruminococcus, and Bifidobacterium species in [38-44]. Conversely, especially Clostridia spp. were found to be increased in children with ASD [42]. The microbiota profiles from healthy siblings of ASD patients are generally found to be an intermediate between the ASD cases and unaffected controls [45]. Associations were found between long-term diets and the presence of different enterotypes.

For example, the presence of Bacteroides spp, a bacterial species is linked to a diet high in fat and animal protein [35,38-44]. Thus, food influences the composition of the microbiome, which affects the production of SCFAs and thereby the intestinal membrane integrity. SCFA-producing bacteria, e.g., Clostridia, Desulfovibrio, and Bacteroides, are increased in feces of children with ASD [39-44]. Clostridium species and Lactobacillus species were increased in the stool of affected children and these bacterial species are known to influence serotonin (5-HT) and catecholamines metabolism with increased 5-HT levels in whole blood and platelets, and together altered function and metabolism of neurotransmitters, and dysfunction of the serotonergic system have been reported to contribute to symptomatology of ASD [5,8,15,19,25,26,35]. 5-HT, thereby, is a potential candidate as a biomarker for ASD. In addition, the presence of Candida was increased two-fold in ASD, showing that the bacterial and fungi microbial communities can mutually influence each other. This might illustrate a general lack in trained immunity or reduced control of overgrowth and would be in support of the hygiene hypothesis connecting bacteria and yeasts [17]. As the intestinal membrane regulates the passage of bacterial compounds and other metabolites, diets can 
have extensive effects in the rest of the body through the Microbiota-Gut-Brain axis (MGB axis).

\section{Diets Influence Brain Functioning by the Microbio- ta-Gut-Brain Axis}

The MGB axis encompasses all signaling pathways between microbiota, gut and brain. This gut-brain axis was already mentioned in the $19^{\text {th }}$ century [26] and elaborated on in the beginning of the $21^{\text {st }}$ century, with the importance of microbiota influencing the communication between gut and brain [28]. Diet can influence these pathways by either changing microbiota composition and/or (bacterial) metabolite production.

Signaling in the MGB axis occurs bidirectional. Signals from the brain affect the motility and integrity of the gut and signals from microbiota and the gut influence brain development and functioning $[28,44]$. Top-down signaling occurs through two different pathways: the nervous system (through the vagus nerve) and the hypothalamic-pituitary-adrenal axis. The Hypothalamic-Pituitary-Adrenal (HPA) axis is an important neuroendocrine system that is involved in stress reactions. Bottom-up signaling is facilitated through three different pathways: the bloodstream, the immune system and the nervous system (including the Enteric Nervous System (ENS) and the vagus nerve) [35]. Here the focus will be on how the GFCF diet changes ASD symptoms from gut to brain.

\section{Relevance of blood transport}

Via the bloodstream signaling occurs when reactive compounds from the gut enter the bloodstream and finally arrive in the brain. In approximately one third of the ASD patients the integrity of the intestinal barrier and BBB is affected [46]. As a consequence, when both the intestinal membrane and the BBB are permeable, pathogenic microbiota, incompletely metabolized (food-derived) peptides, bacterial metabolites and other bacterial compounds can enter the bloodstream, pass the BBB and modulate the development and functioning of the brain $[6,26]$. An example of a bacterial product that is able to pass both the intestinal membrane and the BBB is the SCFA propionate. When propionate reaches the brain it is able to decrease social behavior $[41,44]$.

\section{Immune system in ASD}

The gut microbiota is known to regulate brain activity and homeostasis of microglia through the immune system $[2,12,13,18,41,44]$. Microglia cells are a type of macrophage acting as the first immune defense in the brain. This immune pathway starts with the production of bacterial toxins, e.g., from Clostridia spp., by gut microbiota. Those bacterial toxins evoke an immune response in the gut as well as in the bloodstream and this immune response is accompanied by oxidative stress. Oxidative stress upon the epithelial membranes in turn increases intestinal permeability, resulting in translocation of bacteria into the lamina propria of the mesenteric lymphoid tissue. Subsequently, mucosal immune cells, macrophages and Dendritic Cells (DCs) release pro-inflammatory cytokines (including IL-1 $\beta$, TNF- $\mu$ IL-6 and IL-18).

As described, the altered gut microbiota in ASD by increased species of Lacto bacillus, can modulate the immune response to Candida by providing tryptophan-derived Aryl hydrocarbon Receptor (AhR) ligands. These AhR ligands stimulate the immune system, e.g., ILC3 cells, to produce IL-22, which together with IL-17 inhibits excessive proliferation of Candida in the gut $[44,47]$. Altered gut microbiota could therefore lead to an expansion of Candida in ASD. In addition, bacterial toxins that pass the intestinal membrane can also trigger macrophages and DCs in the blood. Pro-inflammatory cytokines then activate the vagus nerve or reach the brain through the bloodstream. In this way they regulate activity of microglia and functioning of the Central Nervous System (CNS) [2,18,44,47].

\section{The ENS and Nervus Vagus in ASD}

The Enteric Nervous System (ENS) includes also the vagus nerve. The ENS consists of millions of neurons that regulate the motility of the gutin dependently of the brain and the spinal cord [5]. The vagus nerve contains both afferent and efferent nerves that connect the gut with the brain $[5,26,28]$. The afferent nerve fibres sense immune, microbial and nutrient information from the gut and communicate this to the brain. Nerve cells can for example measure the acidity of the intestinal content and start firing when the acidity changes [26]. Neuroactive compounds produced by gut microbiota, including Dopamine (DA), serotonin (5-HT), $\gamma$-Aminobutyric Acid (GABA) and Histamine (His) are well known neuroactive compounds that influence the brain in ASD through the vagus nerve $[5,8,28,35]$.

\section{Gluten and Casein Proteins Affect the Brain via the Bloodstream}

The GFCF diet is an elimination diet, which implies that certain food proteins, like gluten and casein, need to be eliminated from the usual diet and many studies investigated the effects of this dietary intervention on ASD symptoms [48-59]. In the GFCF diet, processed carbohydrates containing gluten are replaced with gluten-free processed carbohydrates generally obtained from rice, sugar, potato starch, tapioca flour, soy, buckwheat flour, etc. These carbohydrates will, however, also be used by the abnormal gut microbiota in ASD patients thereby perpetuating the vicious circle of a damaged leaky gut and peptides occurring in the blood and brain [40]. The relevance of the GFCF diet has likely been overestimated and presented by industry as a general solution for ASD, while this diet is only partially effective as shown in few small sample-sized randomized controlled clinical trials [52].

Yet, the pathways through which the changes in symptoms are obtained are still largely unknown. Both gluten and casein elicit effects on the functionality and the development of the brain and the immune system and nervous system are the best-described modes of action. Reduced pain sensitivity (analgesia) and impaired social behavior are common ASD symptoms that can supposedly be alleviated when following the GFCF diet, probably since derivatives of gluten and casein stimulate the opioid system $[5,6,38,60,61]$. The opioid system consists of three different opioid receptors; $\delta, \kappa$ and $\mu$, all most abundantly present in the brain. Nevertheless, opioid receptors are also found on cells of the digestive, immune, nervous and endocrine system [62]. Opioid ligands consist of endogenous opioids produced inside the body, and exogenous opioids from outside the body (exorphins).

\section{ß-CM7 opioid peptides and DPP4 activity}

In 1999, the role of $\beta$-casomorphin-7 ( $\beta$-CM7 consisting of TyrPro-Phe-Pro-Gly-Pro-Ile) as an exogenous opioid released from $\beta$-casein in cow's milk and was described in relation to autism [63-65]. 
The $\beta$-CM7 peptide is degraded in the GI-tract by the hydrolyzing enzyme dipeptidyl peptidase IV (DPP4; EC 3.4.14.5), a process that is induced by microbiota [65-69].

The family of dipeptidyl peptidases are enzymes able to cleave the prolyl bond of two N-termini thereby cleaving peptides like $\beta$-CM7 (and also circulating hormones, neuro peptides and chemokines like CXCL12) into di- and tri peptides that can be transported across intestinal mucosa. DPP4 is expressed on most organs in the body, including the lung and the GI tract, on epithelial cells, endothelial cells and on immune cells in for example the spleen [70,71]. DPP4 was studied as a dietary protein because it is found in the GI tract as a brush border enzyme. This enzyme is also found on immune cells and therefore coded as CD26 and plays a role in T-cell dependent immune responses while also displaying anti-inflammatory activity [66-68].

The $\beta$-CM7 peptide is known to be a $\mu$-Opioid Receptor (MOR) agonist, and it can also interact with serotonin system, while eliciting comparable effects as the well-known narcotic pain relieving substance morphine [64-65,72-75]. In addition, there are also gluten-derived opioid peptides (exorphins like A4, A5, B4 and B537) that elicit similar effects in the brain through interaction with the $\delta$-opioid receptor [76]. These dietary exorphins do bind with only low affinity for opioid receptors [77].

Additionally, many other dietary proteins might contain amino acid sequences that exhibit antagonist activity on these opioid receptors. DPP4 is able to decrease opioid peptide levels in body fluids, including blood, urine and cerebrospinal fluid [65-68,78]. Consequently, if DPP4 is defective, opioid peptides will not be inactivated and opioid activity in the body rises [66-68]. Both, gluten and casein derived opioid peptides function as agonists of the endogenous opioid system and thereby might alleviate ASD symptoms. A widely adopted theory supporting this mode of action is the opioid excess theory, which was first proposed by Panksepp in 1979 [79].

\section{Excessive Opioid Activity in Patients with Autism Spectrum Disorder}

The opioid excess theory provides an explanation of how excessive absorption of opioid peptides elicits a disruptive effect in the brain and how the GFCF diet can putatively relieve symptoms of ASD $[16,80]$. Under physiological conditions, opioid peptides, including $\beta-\mathrm{CM} 7$, are produced in the gut by the lytic activity of peptidases and part of these opioid peptides can cross, after which they enter the bloodstream $[81,82]$. Tight junctions in the BBB stop the majority of opioid peptides to enter the CNS $[83,84]$. The opioid excess theory hypothesizes that in ASD patients there are multiple alterations that interfere with the formation of opioid peptides and their passing through membranes. These alterations can occur simultaneously and all result in a greater amount of opioid peptides entering the CNS and affecting development and functioning of the brain.

Increased intestinal permeability, results in more opioid peptides in the blood and affected BBB integrity results in excessive amounts of opioid peptides in the brain. These two alterations are often described in literature to be co-occurring with ASD [82-84]. However, abnormally high concentrations of opioid peptides in either plasma or the nervous system of patients with ASD have not consistently been found [81]. In addition, the peptidase enzyme activity can either be decreased due to a defective functioning or a shortage of peptidases. In both cases less opioid peptides are broken down, resulting in elevated levels in the gut, blood and brain [82-84].
Excessive amounts of opioid peptides passing the BBB might influence brain development and functioning by directly binding to opioid receptors. Additionally, these large amounts of opioid peptides saturate peptidase enzymes and not all peptides will be hydrolyzed, pass the BBB, and bind to opioid receptors in the brain [80]. Therefore, gluten and casein, being precursors for opioid peptides, ultimately affect neurotransmission in the brain either directly or indirectly [23]. One obvious and often applied way to diminish opioid activity in the brain is by following the GFCF diet.

\section{Mechanisms of Brain Effects by Casein Derived Opioid Peptide $\beta$-CM7}

\section{$\beta-C M 7$ generated by cow's milk digestion}

Both in vitro and in vivo digestive studies investigated at what stage of the digestion $\beta$-casomorphins were produced from cow's milk [85-88]. $\beta$-casomorphins were found after the in vitro simulation of pancreatic and intestinal digestion, but not after the simulation of stomach digestion. After the consumption of cow's milk in human adults considerable concentrations of $\beta$-CM7 were shown in the gut $[89,90]$. After the formation of $\beta-\mathrm{CM} 7$ in the gut and if $\beta-\mathrm{CM} 7$ is able to cross the intestinal membrane, considerable concentrations of $\beta$-CM7 should be found in the blood and urine. Kost et al., [78] detected $\beta$-CM7 in blood plasma of 1 year olds after cow's milk ingestion, and this finding was confirmed by the study of Sokolov et al., [91] who investigated the urinary $\beta-\mathrm{CM} 7$ concentration.

A significantly higher level of $\beta$-CM7 was found in the urine of children with ASD when compared to controls and an increase in $\beta$-CM7 urinary concentrations was measured when the severity of ASD increased [91]. In contrast, another study did not find an abnormally high $\beta$-CM7 level in the urine of ASD patient, probably due to the small sample size that was used [92]. Consequently, it is likely that $\beta$-CM7 is able to cross the intestinal membrane and reach the BBB through the blood. To show that $\beta$-CM7 affects ASD symptoms through the $\mu$-opioid receptor in the brain, its opioid activity needs to be screened. Teschemacher [73] describes two different tests that can be used to screen the opioid activity: the adenylate cyclase test and the naloxone challenge test. The adenylate cyclase test is a test that investigates binding of opioid agonists to $\mu$-opioid receptors and the subsequent activation of adenylate cyclase. High activity implies that the tested compound induces considerable opioid activity. The naloxone challenge test is an inhibition test in which the high affinity $\mu$-opioid receptor antagonist naloxone is potentially blocked by interaction with an exogenous opioid [64]. Different studies applied both tests and found that $\beta-\mathrm{CM} 7$ induces opioid activity through binding to the $\mu$-opioid receptor [73,74-76].

\section{Opioid activity of $\beta-C M 7$ and ASD behavior}

The passage of $\beta$-CM7 through the BBB and its opioid activity in the brain can be investigated by measuring $\beta-\mathrm{CM} 7$ induced immuno reactivity in different regions (e.g., the nucleus accumbens) in the brain of rats with c-fos. C-Fos is used as a marker protein for neuronal activity [64]. The results showed that $\beta$-CM7 was able to cross the BBB because c-Fos expression was detected in different brain areas upon peripheral administration of $\beta$-CM7. In addition, $\beta$-CM7 activated opioid receptors because c-Fos expression was reduced after pretreatment with naloxone [73,74]. Expression of c-Fos was found in similar brain regions as the regions affected in ASD patients. However, Amaral et al., [5] made use of postmortem 
investigation, as well as structural magnetic resonance imaging methods in humans, and showed other brain regions to be involved in ASD. In rats it was shown that $\beta$-CM7 ultimately triggered ASD-like behavioral changes by dietary administration of $\beta$-CM7 linked to segmented brain activity as shown before [64]. Behavior after administration of $\beta$-CM7 changed from very active and aggressive to significantly reduced responsiveness to sound, a lack of explorative behavior and reduced or absent social interaction, consistent with ASD-like behavior $[9,24,60,79,93,94,95]$.

Taken together, the casein derived opioid peptide $\beta-\mathrm{CM} 7$ was found to trigger ASD-like behavior through the MGB axis. $\beta-C M 7$ is produced in the gut, is able to pass the intestinal membrane and reaches the $\mathrm{BBB}$ through the blood. Thereafter it passes the $\mathrm{BBB}$ and triggers ASD-like behaviors upon binding to the $\mu$-opioid receptor in the brain. $\beta$-CM7 is used as a model to provide insight in the mechanism of action of all gluten and casein derived opioid peptides. Other gluten and casein derived opioid peptides probably trigger ASD symptoms through the bloodstream in a similar fashion.

\section{Effects of the Gluten-Free and Casein-Free Dietary Intervention in ASD}

In 2008, a Cochrane publication could not conduct a systematic review as only two Randomized Controlled Trials (RCT) were available involving programs which eliminated both gluten and casein from the diets of individuals diagnosed with ASD [24,36,48]. It was concluded that exclusion diets are not without cost in terms of inconvenience and extra financial cost and limitations on foods of choice for the affected family member and thus GFCF diet at that time could not recommend as a standard treatment. It was suggested that well-conducted and adequately-powered randomized controlled trials were urgently needed [21].

\section{Short and long term GFCF intervention studies}

Since then, many studies reported on the effects of the GFCF dietary intervention on changes in ASD symptoms [11,22,23,36,46, $49,50,51,54,57]$. Changes in behavioral ASD symptoms, verbal and non-verbal communication and non-neuronal symptoms were investigated in these studies. A distinction is made between long-term studies ( $>6$ months treatment) and short-term studies ( $<6$ months treatment), because the duration of the intervention has an effect on the efficacy of the intervention. Many long-term studies unanimously observed alleviating effects on ASD symptoms and behavioral symptoms in ASD patients in comparison to control subjects. In these studies considerable ameliorations were found in behavioral symptoms such as social isolation and repetitive behaviors [22,23,36,51,57]. Non-neuronal symptoms such as increased intestinal permeability significantly decreased after the GFCF dietary intervention as well. In contrast, results of three short-term studies on behavioral ASD symptoms were not consistent. First, Johnson et al., [50] did not find an improvement of behavioral symptoms during a 3-month intervention period. However, the authors suggested the possibility of alleviating effects if the dietary intervention lasted over a longer period of time. In contrast, Nazni et al., [22] reported improved behavioral ASD symptoms after a intervention of 2 months. Lucarelli et al., [11] conducted a dietary challenge instead of a dietary elimination. They investigated the effects of 8 weeks oral challenge with cow's milk and found increased behavioral ASD symptoms such as social isolation. In summary, outcomes of short-term intervention studies showed variable effects on ASD symptoms.

\section{Different individual GFCF diet responsiveness}

In addition to the duration of the intervention, the efficacy of the GFCF dietary intervention depends on the age of the patient. Whiteley et al., [55] conducted the Scan Brit study; a randomized controlled trial of 2 years and found significant changes in symptoms in ASD patients compared to the control group. However, results showed considerable variability in individual responses to the GFCF diet. In continuation on the Scan Brit study, Pedersen et al., [53] found that age was the most important predictor for the difference in response between participants. In young children between 7 and 9 years old the GFCF intervention effectively alleviated ASD symptoms. In addition, a third study reported increased alleviation of ASD symptoms in children compared to adults [58]. These studies indicate that at young age the GFCF diet is most effective.

\section{Allergy and/or intolerance linked to ASD}

Classical food allergies result from strong IgE-mediated immune responses to a food, and the resulting inflammation can involve multiple body systems $[18,41,44]$. Typically, this results in the release of histamine and other inflammatory mediators with typical allergy symptoms as the result. Food intolerances are associated with a non-immune response to a component of a food, e.g., inability to digest lactose (milk sugar). This reaction can also cause GI distress including stomach pain, bloating and diarrhea. Both these reactions can also occur in children suffering from ASD who may not be able to communicate or fully understand or explain their discomfort. Parents often list milk, gluten, soy, corn and egg proteins as the primary offenders. Besides, in ASD children increasingly symptoms are observed relating to eosinophilic esophagitis that involves a mixed IgE and non-IgE response with a resulting inflammation that produces changes in the cells lining the esophagus. Avoidance of the offending foods allows the resolving of the inflammation of the esophagus and the rest of the GI tract. This type of delayed inflammatory response can affect sleep, focus and attention and can also worsen irritability, hyperactivity and repetitive behaviors in children with autism.

The presence of gluten and casein allergies or intolerances will influence the individual responsiveness to the GFCF dietary intervention. Mulloy et al., [16] reviewed 14 studies on the effects of GFCF diets in ASD patients with or without allergy and only limited evidence for the efficacy of the GFCF diet was found. These authors advised to only implement the GFCF diet if the patient is diagnosed with a gluten and/or casein allergy or intolerance. All in all, duration of the intervention and age are two important predictors of the efficacy of the GFCF diet in people with ASD. In case of a long-term dietary intervention at young age, the efficacy is predicted to be highest. Moreover, if the ASD patient is diagnosed with a gluten and/or casein allergy or intolerance the GFCF diet might be recommended [32].

\section{Risks associated with GFCF diets in ASD patients}

Considering the GFCF diet as potential therapy for people with ASD, putative negative effects of following the GFCF diet should also be taken into account. Several concerns might exist, including the risk of nutritional deficiencies due to a decreased protein intake $[2,12,74]$. However, Johnson et al., [50] conducted a study of 3 months on the effects of the GFCF diet in young children with ASD. No nutritional deficiencies were observed in the intervention group compared to the control group. A cross-sectional study examined dietary supplement use and micronutrient intake that are commonly 
used in children with ASD. Only few children needed most of the micronutrients while in many others excess intake of vitamin A, folate, and zinc was often observed. However, even when supplements were used, careful attention should be given to adequacy of vitamin $\mathrm{D}$, calcium, potassium, pantothenic acid, and choline intake [33]. Another concern upon starting an elimination diet is the increased risk of social rejection. Following the GFCF diet hampers people with ASD to integrate and socialize with others [96]. Also, implementation of the GFCF diet might be difficult, as people with ASD are known to often have already heavily restricted eating behaviors [7].

All in all, many studies investigated the positive effects as well as the downsides of the GFCF dietary intervention. The outcomes of these studies are not unanimous which can be explained by the differences in duration of the interventions. Variability in individual response to the GFCF diet can be explained by age of the patient. Moreover, if the ASD patient is diagnosed with gluten and casein allergy or intolerance, the GFCF diet probably is effective too. In addition, there are other factors that are thought to explain individual differences in response to the GFCF dietary intervention including intestinal permeability, BBB integrity and DPP4 enzyme activity.

\section{Role of Intestinal Permeability, Blood Brain Barri- er Integrity and DPP4 Enzyme Activity in ASD}

Other individual differences that modulate the response to the GFCF diet include an increased intestinal permeability, which is seen in a substantial proportion of children with ASD, a more permeable BBB and a decreased DPP4 enzyme activity [74]. Because of these alterations toxic bacterial products, pathogenic bacteria and opioid peptides enter the bloodstream and the brain more easily, influencing brain functioning.

\section{Altered intestinal permeability in people with autism spectrum disorder}

As mentioned before, the intestinal permeability is one of the factors that determine whether opioid peptides can enter the bloodstream. There are two non-invasive tests to measure intestinal permeability. The Intestinal Permeability Test (IPT) measures the ability of two sugars (lactulose and mannitol) to penetrate the intestinal membrane. The better they are able to penetrate, the more permeable the intestinal membrane [46]. Alternatively, the urinary peptide concentration can be used as a marker for the intestinal permeability. The more peptides are found in the urine, the more permeable the intestinal membrane [97]. It is worth noting that the urinary peptide concentration is a marker for functioning of peptidases such as DPP4 as well. Concerning peptidase activity, an increase of the urinary peptide concentration implies a decreased peptidase activity [98].

With regard to the IPT, several studies found an increased intestinal permeability in $25-43 \%$ of autistic patients in contrast to $0 \%$ $4.8 \%$ in the control group $[46,82,99]$. In addition, the GFCF dietary intervention study measured that the intestinal permeability returned to physiological limits after the dietary intervention [46]. Two studies measured the urinary peptide concentration in people with ASD to assess their intestinal permeability. In both studies significantly higher levels of urinary peptides were found in autistic subjects when compared to control subjects $[91,98]$. Whether the higher concentration of urinary peptides was caused by an increased intestinal permeability and/or by decreased peptidase activity remains uncertain, because IPTs were not conducted in these studies.
Besides functional testing for intestinal permeability, intestinal biopsies can also be used. Fiorentino et al., [84] investigated expression of genes related to epithelial barrier-forming and pore-forming in duodenal biopsies of ASD patients. Decreased expression of at least one barrier-forming gene was found in $75 \%$ of all patients, while in $66 \%$ of all patients an increased level of pore-forming genes was detected. These results indicate that an increased intestinal permeability is possibly caused by alterations in genes related to barrier integrity in people with ASD.

\section{Blood brain barrier integrity in people with autism spec- trum disorder}

BBB integrity could be altered in people with ASD and was obtained in several studies $[8,83,100]$. Fiorentino et al., [84] performed a postmortem study to investigate BBB integrity in ASD patients. Gene expression levels as well as protein levels of major components involved in the regulation of BBB integrity were measured. Altered expression of these genes was found, possibly reflecting an impaired BBB integrity. In line with these findings de Theije et al., [8] concluded that $\mathrm{BBB}$ integrity is decreased in autistic patients by combining the results of two studies. In the first study Emanuele et al., [100] found an association between increased plasma levels of Lipopolysaccharide (LPS), present in the membrane of gram-negative bacteria, and autistic patients. The second study was a postmortem study that investigated the functional effects of LPS on BBB permeability on a protein related to Alzheimer's disease. They found that LPS increases BBB permeability to this protein [83]. Taken together, BBB permeability is likely to be increased in ASD patients, facilitating the passage of opioid peptides and other compounds from blood to brain.

\section{DPP4 enzyme activity in people with autism spectrum disorder}

DPP4 enzyme activity is altered in ASD patients and thus opioid peptide levels in the blood, urine and brain rise in the case of decreased DPP4 activity. DPP4 is thus able to decrease endogenous opioid peptide levels as well as exogenous opioid peptide levels in body fluids. In addition to DPP4 activity, these concentrations reflect intestinal permeability. Several studies found a significantly higher level of blood plasma $\beta$-endorphin, an endogenous opioid peptide in autistic subjects when compared to controls $[76,94,95]$ and plasma $\beta$-endorphin was positively associated with the severity of ASD symptoms [95]. Moreover, an increase of $\beta$-endorphin levels in the cerebrospinal fluid was reported [79]. Elevated levels of other endogenous opioid peptides were also found in the peripheral blood of ASD patients [94].

There are multiple explanations for the rise of endogenous opioids in body fluids. It might be caused by an excessive amount of exogenous opioids (such as $\beta$-CM7) that saturates DPP4 enzymes so that they cannot degrade endogenous opioids. Also, the rise in endogenous opioids can be explained by a defect in the DPP4 enzyme [97] although this is debated by others [67], or DPPIV gene mutations [65]. In addition, decreased expression of DPP4 possibly explains the rise of endogenous opioids in body fluids. In line with this explanation Hunter et al., [67] detected less cells that expressed DPP4 in autistic children when compared to the control group. An increase in intestinal permeability was found in approximately a third of all patients with ASD $[46,82,99]$. Moreover, BBB integrity is likely to be decreased in ASD patients. Furthermore, abnormally high levels of 
$\beta$-endorphin and other endogenous opioids were found in body fluids of ASD patients. This can be explained by an increased intestinal permeability and/or a decreased DPP4 enzyme activity.

\section{Urinary peptide analysis in ASD}

Urinary investigation revealed the presence of distinct urinary profiles common peaks in the urines of people with autism. Opioid peptides derived from dairy and cereal products had been identified in the urine of children with autism. In contrast to previously mentioned studies, results of studies that investigated levels of urinary opioid peptides in ASD were ambiguous $[67,79,91,101]$. One study found elevated levels of urinary exogenous opioids in autistic children [77,91]. In contrast, Cass et al., [102] and Hunter et al., [67] did not detect differences in urinary opioid peptide concentrations between ASD subjects and controls. However, Shattock et al., [103] discovered important flaws in the chemical analysis that were used to determine the opioid peptide levels in the study of Hunter et al., [67]. In urine of ASD children several exorphins could be detected in which the length of the peptides varied between individuals [98]. The results from a GFCF intervention have shown significant improvements in the behavioral and cognitive functioning of participants, with regression reported following the suspension of the diet. Similar changes in the pathological urine patterns and levels of peptides have also been demonstrated in children with ASD on dietary intervention. However, laboratory tests often failed to detect normalization of urinary peptides even when clinical improvement was indicated [56].

\section{Critical Appraisal}

The most common feature underlying the ASD condition is the inflammation in the brain which could be caused by different environmental toxic compounds, infections, and co-morbidities in individuals that are genetically susceptible to the development of ASD [104]. The well accepted bidirectional communication between the brain and the gut microbiome has sparked the interest in altered gut-brain interactions in ASD patients. These interactions not only contribute to ASD patho physiology but also to disease burden and open possibilities for novel treatment options in ASD. Gut-microbiome directed therapies, including fecal transplantation, may eventually translate into clinical improvement [105]. However, there is considerable controversies concerning published findings and future mechanistic research is certainly implicated [106-109].

The GFCF diet is the most prevalently followed diet in ASD patients. However, the GFCF diet has not been shown to be effective in all patients, which in general results in negative advice to start this dietary intervention [16]. ASD is a heterogeneous condition, which implies many personal differences that explain the varying responsiveness to the GFCF diet [56]. The main challenge is to use the GFCF diet efficiently, which means that only patients that would probably benefit from it should start the dietary intervention. This requires improved knowledge about factors that influence the responsiveness and underlying mechanisms of action by which the diet elicit effects on the brain.

Possible explanations for the variable individual responsiveness to the GFCF diet in ASD patients include intestinal membrane permeability, BBB integrity, DPP4 enzyme activity, duration of the intervention and age. The most important factor that is related to the effectiveness of the diet is intestinal membrane permeability as this was found in approximately one third of all ASD patients $[46,82,99]$.
IPTs are useful tests to measure intestinal permeability, as they are non-invasive [46]. However, studies that measured urinary peptide concentrations were not sure whether an increase in these concentrations was caused by an increased intestinal permeability and/or decreased peptidase activity. Therefore, primarily the IPT emerged as a reliable test to predict the responsiveness to the GFCF diet. This is in line with Whiteley et al., [80], who mentioned the relevance of measuring intestinal membrane permeability to investigate best- and non-responders to the GFCF dietary intervention.

BBB integrity largely determines whether the GFCF diet is effective as increased BBB permeability allows more opioid peptides to enter the brain and trigger ASD symptoms [80]. De Theije et al., [8] concluded that BBB integrity was decreased in ASD patients but this conclusion was based on combining two different studies $[83,100]$. These studies found an increased level of LPS in autistic patients and an increased BBB permeability for an Alzheimer related protein after injection of LPS.

Fiorentino et al., [84] found increased expression levels of two genes involved in barrier formation in ASD patients which seems contradictory with the conclusion of de Theije et al., [8] although a compensatory mechanism for a decreased BBB integrity cannot be excluded [84]. An important limitation concerning investigation of the BBB integrity is the lack of direct measurement of the BBB permeability to opioid peptides. Measuring opioid peptide concentrations in the brain of living human is not feasible [84]. Therefore, BBB integrity cannot be used as a predictive factor of responsiveness to the GFCF diet in ASD patients.

DPP4 activity determines the amount of opioid peptides in body fluids but these levels reflect both DPP4 activity and intestinal permeability. Nevertheless, the amount of DPP4 enzymes is decreased in ASD patients, resulting in a higher amount of opioid peptides in the body $[76,94,95]$. Further research should use the combination of measuring opioid peptide concentrations in body fluids and an IPT. In case of an intestinal permeability within physiological limits, it is likely that elevated concentrations of opioid peptides are caused by decreased DPP4 activity.

The duration of the GFCF intervention should be taken into account as all studies that showed significant alleviating effects performed the dietary intervention of 6 months or more [22,23,36,51,57]. Therefore it is important that patients are well informed about the delay in effects of the GFCF diet.

The age of the patient at which the intervention starts is of importance as intervention is more effective in children when compared to adults [58]. Best outcomes were obtained if children start with the diet between age 7 and 9 [53].

The mode of action through which $\beta$-CM7 elicits effects on the brain through the bloodstream starts with the digestion of cow's milk into $\beta$-CM7 in the gut [89] and subsequent crossing the intestinal membrane in rats $[78,91]$. No human studies were conducted that investigated if $\beta$-CM7 passed the BBB and induces opioid activity in the brain [11].

Discrepancies were observed between the brain areas where opioid activity was measured in rats [64] and the brain areas related to ASD in humans [5]. However, structural and biochemical changes in the brain caused by (the elimination of) gluten and casein remain largely unexplored. 
Regarding effect studies of the GFCF diet in people with ASD, there is a lack of well-designed randomized placebo-controlled intervention studies. Often reliability is lacking due to a small sample size [11], short duration of the intervention [11,22,50,54], lack of placebo [22], single blinding [36] and a non-randomized methodology [22]. Consequently, reviews on the effects of the diet in ASD patients were contradictory and inconclusive $[16,51]$.

Johnson et al., [50] did not find any nutritional deficiencies in ASD patients after the GCFC intervention. However, the presence of nutritional deficiencies was investigated after an intervention period of 3 months. Possibly, nutritional deficiencies might evolve during a longer intervention period.

The various characteristics of ASD, as discussed here, are summarized in figure 1 . These mechanisms provide explanations for the putative positive effects of GFCF diets to mitigate symptoms of ASD in susceptible individuals.

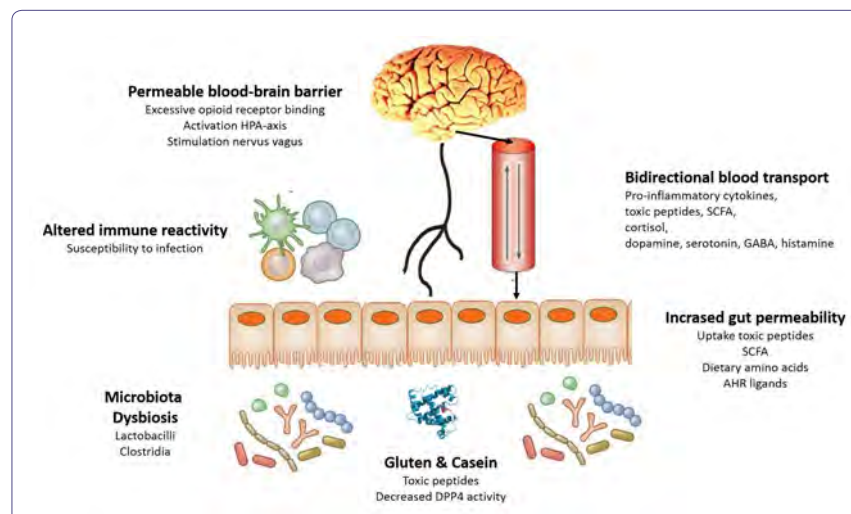

Figure 1: Processes characteristic for ASD that are all inhibited by a Gluten-Free And Casein-Free (GFCF) diet and potentially block symptoms of ASD.

DPP4: Dipeptidyl peptidase 4; GABA: $\gamma$-aminobutyric acid; HPA: Hypothalamus Pituitary Adrenal Cortex; SCFA: Short-Chain Fatty Acids.

\section{Conclusion}

Several factors were found to influence the individual responsiveness to the GFCF dietary intervention in ASD patients. A higher responsiveness is expected if the intervention starts between age 7 and 9 and if the GFCF diet is followed for at least 6 months. Moreover, increased intestinal permeability, decreased BBB integrity and decreased DPP4 activity make ASD patients more prone to consequences of gluten and casein exposure, and therefore more responsive to the GFCF diet. Measuring intestinal permeability with an IPT as a predictor of responsiveness to the GFCF dietary intervention is promising. However, further research to prove the association between an increased intestinal permeability and higher responsiveness to the GFCF diet is recommended. Finally, it is probable that gluten and casein elicit effects on the brain through the bloodstream, showing the importance of the MGB axis in ASD. Notwithstanding, how opioid peptides after entering the brain are able to trigger ASD-like symptoms remains largely unknown. Here we focused on effects the GFCF diet might have through the bloodstream. However, gluten and casein derived opioid peptides also act through the immune system and the nervous system and these systems provide additional explanations for individual differences in response to the GFCF diet in ASD.

\section{References}

1. Carrington SJ, Kent RG, Maljaars J, Le Couteur A, Gould J, et al. (2014) DSM-5 Autism Spectrum Disorder: In search of essential behaviours for diagnosis. Research in Autism Spectrum Disorders 8: 701-715.

2. Noriega DB, Savelkoul HF (2014) Immune dysregulation in autism spectrum disorder. Eur J Pediatr 173: 33-43.

3. WHO (2017) Autism spectrum disorders. WHO, Geneva, Switzerland.

4. Piskorz-Ogórek K, Ogórek S, Cieślińska A, Kostyra E (2015) Autism in Poland in comparison to other countries. Polish Annals of Medicine 22: $35-40$.

5. Amaral DG, Schumann CM, Nordahl CW (2008) Neuroanatomy of autism. Trends Neurosci 31: 137-145.

6. McConachie H, Diggle T (2007) Parent implemented early intervention for young children with autism spectrum disorder: A systematic review. J Eval Clin Pract 13: 120-129.

7. Cermak SA, Curtin C, Bandini LG (2010) Food Selectivity and Sensory Sensitivity in Children with Autism Spectrum Disorders. YJADA 110: 238-246.

8. De Theije CGM, Wu J, Da Silva SL, Kamphuis PJ, Garssen J, et al. (2011) Pathways underlying the gut-to-brain connection in autism spectrum disorders as future targets for disease management. Eur J Pharmacol 668: 70-80.

9. Čupić B, Hranilovic D, Jernej B, Gabrilovac J (2012) Association study of genes regulating opioid system in autism. Psychiatry Res 198: 169-170.

10. Lyall K, Ashwood P, Van de Water J, Hertz-Picciotto I (2014) Maternal Immune-Mediated Conditions, Autism Spectrum Disorders, and Developmental Delay. J Autism Dev Disord 44: 1546-1555.

11. Lucarelli S, Frediani T, Zingoni AM, Ferruzzi F, Giardini O, et al. (1995) Food allergy and infantile autism. Panminerva Med 37: 137-141.

12. Goines PE, Ashwood P (2013) Cytokine dysregulation in autism spectrum disorders (ASD): Possible role of the environment. Neurotoxicol Teratol 36: $67-81$.

13. Gibney SM, Drexhage HA (2013) Evidence for a dysregulated immune system in the etiology of psychiatric disorders. J Neuroinmmune Pharmacol 8: 900-920.

14. Chen MH, Su TP, Chen YS, Hsu JW, Huang KL, et al. (2013) Comorbidity of allergic and autoimmune diseases in patients with autism spectrum disorder: A nationwide population-based study. Research in Autism Spectrum Disorders 7: 205-212.

15. Zerbo O, Leong A, Barcellos L, Bernal P, Fireman B, et al. (2015) Immune Mediated Conditions in Autism Spectrum Disorders. Brain Behav Immun 46: 232-236.

16. Mulloy A, Lang R, O’Reilly M, Sigafoos J, Lancioni G, et al. (2010) Gluten-free and casein-free diets in the treatment of autism spectrum disorders: A systematic review. Research in Autism Spectrum Disorders 4: 328-339.

17. Strati F, Cavalieri D, Albanese D, De Felice C, Donati C, et al. (2017) New evidences on the altered gut microbiota in autism spectrum disorders. Microbiome 5: 24

18. Akintude ME, Rose M, Krakowiak P, Heuer L, Ashwood P, et al. (2015) Increased production of IL-17 in children with autism spectrum disorders and co-morbid asthma. J Neuroimmunol 286: 33-41.

19. Li Q, Han Y, Dy ABC, Hagerman RJ (2017) The Gut Microbiota and Autism Spectrum Disorders. Front Cell Neurosci 11: 120.

20. Green VA, Pituch KA, Itchon J, Choi A, O’Reilly M, et al. (2006) Internet survey of treatments used by parents of children with autism. Res Dev Disabil 27: 70-84. 
21. Millward C, Ferriter M, Calver S, Connell-Jones G (2008) Gluten-and casein-free diets for autistic spectrum disorder. Cochrane Database Syst Rev 16.

22. Nazni P, Wesely EG, Nishadevi V (2008) Impact of Casein and Gluten Free Dietary Intervention on Selected Autistic Children. Iran J Pediatr 18: 244-250.

23. Pennesi CM, Klein LC (2012) Effectiveness of the gluten-free, casein-free diet for children diagnosed with autism spectrum disorder: Based on parental report. Nutr Neurosci 15: 85-91.

24. Knivsberg AM, Reichelt KL, Hoien T, Nodland M (2003) Effect of dietary intervention on autistic behavior. Focus Autism Other Developmental Disablities 18: 248-257

25. Reichelt KL, Knivsberg AM, Lind G, Nodland M (1991) Probable aetiology and possible treatment of childhood autism. Brain Dysfunction 4 308-319.

26. Bauer KC, Huus KE, Finlay BB (2016) Microbes and the mind: emerging hallmarks of the gut microbiota-brain axis. Cell Microbiol 18: 632-644.

27. Strati F, Cavalieri D, Albanese D, De Felice C, Donati C, et al. (2017) New evidences on the altered gut microbiota in autism spectrum disorders. Microbiome 5: 24

28. Cryan JF, Dinan TG (2012) Mind-altering microorganisms: the impact of the gut microbiota on brain and behaviour. Nat Rev Neurosci 13: 701-712.

29. Li Q, Zhou JM (2016) The microbiota-gut-brain axis and its potential therapeutic role in autism spectrum disorder. Neuroscience 324: 131-139.

30. De Vos WM, de Vos EA (2012) Role of the intestinal microbiome in health and disease: from correlation to causation. Nutr Rev 70: 45-56.

31. Adams JB, Johansen LJ, Powell LD, Quig D, Rubin RA (2011) Gastrointestinal flora and gastrointestinal status in children with autism-comparisons to typical children and correlation with autism severity. BMC Gastroenterol 11: 22.

32. Srinivasan P (2009) A review of dietary interventions in autism. Ann Clin Psychiatry 21: 237-247.

33. Stewart PA, Hyman SL, Schmidt BL, Macklin EA, Reynolds A, et al (2015) Dietary supplementation in children with autism spectrum disorders: common, insufficient, and excessive. J Acad Nutr Diet 115: 12371248 .

34. Foster JA, Mcvey Neufeld KA (2013) Gut-brain axis: how the microbiome influences anxiety and depression. Trends Neurosci 36: 305-312.

35. Bercik P, Collins SM, Verdu EF (2012) Microbes and the gut-brain axis. Neurogastroenterol Motil 24: 405-413

36. Knivsberg AM, Reichelt KL, Hoien T, Nodland M (2002) A randomized controlled study of dietary intervention in autistic syndromes. Nutr Neurosci 5: 251-261.

37. Fiedorowicz E, Markiewicz LH, Sidor K, Świątecka D, Cieślińska A, et al. (2016) The influence of breast milk and infant formulae hydrolysates on bacterial adhesion and Caco-2 cells functioning. Food Research International 89: 679-688.

38. Liu X, Cao S, Zhang X (2015) Modulation of gut microbiota-brain axis by probiotics, prebiotics, and diet. J Agric Food Chem 63: 7885-7895.

39. Ding HT, Tauer Y, Walkup JT (2017) Gut microbiota and autism: key concepts and findings. J Autism Dev Disord 47: 480-489.

40. De Angelis M, Francavilla R, Piccolo M, De Giacomo A, Gobbetti M (2015) Autism spectrum disorders and intestinal microbiota. Gut Microbes 6: 207-213.

41. Hsiao EY, McBride SW, Hsien S, Sharon G, Hyde ER, et al. (2013) The microbiota modulates gut physiology and behavioral abnormalities associated with autism. Cell 155: 1451-1463.
42. Kang DW, Park JG, Ilhan ZE, Wallstrom G, Labaer J, et al. (2013) Reduced incidence of Prevotella and other fermenters in intestinal microflora of autistic children. PLoS One 8: 68322.

43. Rosenfeld CS (2015) Microbiome disturbances and autism spectrum disorders. Drug Metab Dispos 43: 1557-1571.

44. Rooks MG, Garrett WS (2016) Gut microbiota, metabolites and host immunity. Nat Rev Immunol 16: 341-352.

45. Louis P (2012) Does the human gut microbiota contribute to the etiology of autism spectrum disorder? Dig Dis Sci 57: 1987-1989.

46. de Magistris L, Familiari V, Pascotto A, Sapone A, Frolli A, et al. (2010) Alterations of the Intestinal Barrier in Patients With Autism Spectrum Disorders and in Their First-degree Relatives. J Pediatr Gastroenterol Nutr 51: $418-424$

47. Zelante T, Iannitti RG, Cunha C, De Luca A, Giovannini G, et al. (2013) Tryptophan catabolites from microbiota engage aryl hydrocarbon receptor and balance mucosal reactivity via interleukin-22. Immunity 39: 372-385.

48. Elder JH, Shanker M, Shuster J, Theriaque D, Burns S, et al. (2006) The gluten-free, casein-free diet in autism: Results of a preliminary double blind clinical trial. J Autism Dev Disord 36: 413-420.

49. Hsu CL, Lin DCY, Chen CL, Wang CM, Wong MK (2009) The Effects of A Gluten and Casein-free Diet in Children with Autism: A Case Report Chang Gung Med J 32: 459-465.

50. Johnson CR, Handen BL, Zimmer M, Sacco K, Turner K (2011) Effects of Gluten Free / Casein Free Diet in Young Children with Autism: A Pilo Study. Journal of Developmental and Physical Disabilities 23: 213-225.

51. Lange KW, Hauser J, Reissmann A (2015) Gluten-free and casein-free diets in the therapy of autism. Curr Opin Clin Nutr Metab Care 18: 572-575.

52. Mar1'-Bauset S, Zazpe I, Mari-Sanchis A, Llopis-Gonza'lez A, Morales-Sua'rez-Varela M (2014) Evidence of the Gluten-Free and Casein-Free Diet in Autism Spectrum Disorders: A Systematic Review. J Child Neurol 29: 1718-1727.

53. Pedersen L, Parlar S, Kvist K, Whiteley P, Shattock P (2014) Data mining the ScanBrit study of a gluten- and casein-free dietary intervention for children with autism spectrum disorders: Behavioural and psychometric measures of dietary response. Nutr Neurosci1 7: 207-213.

54. Seung H, Rogalski Y, Shankar M, Elder J (2007) The gluten- and casein-free diet and autism: communication outcomes from a preliminary double-blind clinical trial. J Med Speech Lang Pathol 15: 337-345.

55. Whiteley P, Haracopos D, Knivsberg AM, Reichelt KL, Parlar S, et al (2010) The ScanBrit randomised, controlled, single-blind study of a gluten- and casein-free dietary intervention for children with autism spectrum disorders. Nutr Neurosci 13: 87-100.

56. Whiteley P, Rodgers J, Savery D, Shattock P (1999) A gluten-free diet as an intervention for autism and associated spectrum disorders: preliminary findings. Autism 3: 45-65.

57. Whiteley P, Shattock P, Carr K, Hooper M, Todd L (2010) How Could a Gluten- and Casein-Free Diet Ameliorate Symptoms Associated with Autism Spectrum Conditions? Autism Insights 2: 39.

58. Whiteley P, Shattock P, Knivsberg AM, Seim A, Reichelt KL, et al. (2012) Gluten- and casein-free dietary intervention for autism spectrum conditions. Front Hum Neurosci 6: 344.

59. Zhang J, Mayton MR, Wheeler JJ (2013) Effectiveness of Gluten-Free and Casein- Free Diets for Individuals with Autism Spectrum Disorders: An Evidence-Based Research Synthesis. Education and Training in Autism and Developmental Disabilities 48: 276-287.

60. Machin AJ, Dunbar RIM (2011) The brain opioid theory of social attachment: a review of the evidence. Behaviour 148: 985-1025. 
61. Sienkiewicz-Szłapka E, Jarmołowska B, Krawczuk S, Kostyra E, Kostyra $\mathrm{H}$ (2009) Transport of bovine milk-derived opioid peptides across a Caco2 monolayer. International Dairy Journal 19: 252-257.

62. Silva SV, Malcata FX (2005) Caseins as source of bioactive peptides. International Dairy Journal 15: 1-15.

63. Sun Z, Cade JR (1999) A Peptide Found in Schizophrenia and Autism Causes Behavioral Changes in Rats. Autism 3: 85-95.

64. Sun Z, Cade RJ, Fregly MJ, Malcolm Privette R (1999) $\beta$-Casomorphin induces Fos-like immunoreactivity in discrete brain regions relevant to schizoprenia and autism. Autism 3: 67-83.

65. Cieślińska A, Sienkiewicz-Szłapka E, Wasilewska J, Fiedorowicz E, Chwała B, et al. (2015) Influence of candidate polymorphisms on the dipeptidyl peptidase IV and $\beta$-opioid receptor genes expression in aspect of the $\beta$-casomorphin-7 modulation functions in autism. Peptides 65: 6-11.

66. Cordero OJ, Salgado FJ, Nogueira M (2009) On the origin of the serum CD26 and its altered concentration in cancer patients. Cancer Immunol Imunnother 58: 1723-1747.

67. Hunter LC, O’Hare A, Herron WJ, Fisher LA, Jones GE (2003) Opioid peptides and dipeptidyl peptidase in autism. Dev Med Child Neurol 45 : 121-128.

68. Matteucci E, Giampietro O (2009) Dipeptidyl Peptidase-4 (CD26): Knowing the function before inhibiting the enzyme. Curr Med Chem 16: 2943 2951.

69. Kamiński S, Cieślińska A, Kostyra E (2007) Polymorphism of bovine beta-casein and its potential effect on human health. J Appl Genet 48: 189198.

70. Lambeir AM, Durinx C, Scharpé S, De Meester I (2003) Dipeptidyl-peptidase IV from bench to bedside: an update on structural properties, functions, and clinical aspects of the enzyme DPP IV. Crit Rev Clin Lab Sci 40: 209-94.

71. Engel M, Hoffmann T, Wagner L, Wermann M, Heiser U, et al. (2003) The crystal structure of dipeptidyl peptidase IV (CD26) reveals its functional regulation and enzymatic mechanism. Proc Natl Acad Sci U S A 100: 5063-5068.

72. Fiedorowicz E, Jarmołowska B, Iwan M, Kostyra E, Obuchowicz R, et al. (2010) The influence of -opioid receptor agonist and antagonist peptides on Peripheral Blood Mononuclear Cells (PBMCs). Peptides 32: 707-712.

73. Teschemacher H (2003) Opioid Receptor Ligands Derived from Food Proteins. Curr Pharm Des 9: 1331-1344.

74. Chang KJ, Fu Su Y, Brent DA, ChangS JK (1985) Isolation of a Specific p-Opiate Receptor Peptide, Morphiceptin, from an Enzymatic Digest of Milk Proteins. The Journal of Biological Chemistry 260: 9706-9712.

75. Sokolov OY, Pryanikova NA, Kost NV, Zolotarev YA, Ryukert EN, et al. (2005) Reactions between beta-casomorphins-7 and 5-HT2-serotonin receptors. Bull Exp Biol Med 140: 582-584.

76. Fukudome S, Jinsmaa Y, Matsukawa T, Sasaki R, Yoshikawa M (1997) Release of opioid peptides, gluten exorphins by the action of pancreatic elastase. FEBS Lett 412: 475-479.

77. Nongonierma AB, FitzGerald RJ (2015) The scientific evidence for the role of milk protein-derived bioactive peptides in humans: A Review. Journal of Functional Foods 17: 640-656.

78. Kost NV, Sokolov OY, Kurasova OB, Dmitriev AD, Tarakanova JN, et al (2009) $\beta$-Casomorphins-7 in infants on different type of feeding and different levels of psychomotor development. Peptides 30: 1854-1860.

79. Panksepp J (1979) A neurochemical theory on autism. Trends in Neurosciences 2: 174-177.
80. Whiteley P, Shattock P (2002) Biochemical aspects in autism spectrum disorders: updating the opioid-excess theory and presenting new opportunities for biomedical intervention. Expert Opin Ther Targets 6: 175-183.

81. Lennernäs H (2007) Intestinal permeability and its relevance for absorption and elimination. Xenobiotica 37: 1015-1051.

82. D’Eufemia P, Celli M, Finocchiaro R, Pacifico L, Viozzi L, et al. (1996) Abnormal intestinal permeability in children with autism. Acta Paediatr 85: 1076-1079.

83. Jaeger LB, Dohgu S, Sultana R, Lynch JL, Owen JB, et al. (2009) Lipopolysaccharide alters the blood-brain barrier transport of amyloid $\mathrm{b}$ protein: A mechanism for inflammation in the progression of Alzheimer's disease. Brain Behav Immun 23: 507-517.

84. Fiorentino M, Sapone A, Senger S, Camhi SS, Kadzielski SM, et al. (2016) Blood-brain barrier and intestinal epithelial barrier alterations in autism spectrum disorders. Mol Autism 7: 49.

85. Cieślińska A, Kamiński S, Kostyra E, Sienkiewicz-Szłapka E (2007) Beta-casomorphin 7 in raw and hydrolyzed milk derived from cows of alternative $\beta$-casein genotypes. Milchwissenschaft 62: 125-127.

86. Cieślińska A, Kostyra E, Kostyra H, Oleński K, Fiedorowicz E, et al. (2012) Milk from cows of different $\beta$-casein genotypes as a source of $\beta$-casomorphin-7. Int J Food Sci Nutr 63: 426-430.

87. Jinsmaa Y, Yoshikawa M (1999) Enzymatic release of neocasomorphin and beta-casomorphin from bovine beta-casein. Peptides 20: 957-962

88. Hartwig A, Teschemacher H, Lehmann W, Gauly M, Erhardt G (1997) Influence of genetic polymorphisms in bovine milk on the occurrence of bioactive peptides. In: Milk protein polymorphism. Proceedings of the IDF Seminar held in Palmerston North, New Zealand. International Dairy Federation, Belgium. Pg no: 459-460.

89. Svedberg J, Haas JDE, Leimenstoll G, Paul F, Teschemacher H (1985) Demonstration of $\beta$-casomorphin immunoreactive materials in in vitro digests of bovine milk and in small intestine contents after bovine milk ingestion in adult humans. Peptides 6: 825-830.

90. Ho S, Woodford K, Kukuljan S, Pal S (2014) Comparative effects of A1 versus A2 beta-casein on gastrointestinal measures: a blinded randomised cross-over pilot study. Eur J Clin Nutr 68: 994-1000.

91. Sokolov O, Kost N, Andreeva O, Korneeva E, Meshavkin V, et al. (2014) Autistic children display elevated urine levels of bovine casomorphin-7 immunoreactivity. Peptides 56: 68-71.

92. Dettmer K, Hanna D, Whetstone P, Hansen R, Hammock BD (2007) Autism and urinary exogenous neuropeptides: development of an on-line SPE-HPLC tandem mass spectrometry method to test the opioid excess theory. Anal Bioanal Chem 388: 1643-1651.

93. Bell SJ, Grochoski GT, Clarke AJ (2006) Health implications of milk containing beta-casein with the A2 genetic variant. Crit Rev Food Sci Nutr 46: 93-100.

94. Nelson KB, Grether JK, Croen LA, Dambrosia JM, Dickens BF, et al (2001) Neuropeptides and neurotrophins in neonatal blood of children with autism or mental retardation. Ann Neurol 49: 597-606.

95. Tordjman S, Anderson GM, Botbol M, Brailly-Tabard S, Perez-Diaz F, et al. (2009) Pain reactivity and plasma beta-endorphin in children and adolescents with autistic disorder. PLoS One 4: 5289.

96. Lázaro CP, Pondé MP, Rodrigues LEA, Lázaro CP, Pondé MP (2016) Opioid peptides and gastrointestinal symptoms in autism spectrum disorders Revista Brasileira de Psiquiatria 38.

97. Reichelt KL, Knivsberg AM (2003) Can the pathophysiology of autism be explained by the nature of the discovered urine peptides? Nutr Neurosci 6: $19-28$. 
98. Reichelt KL, Tveiten D, Knivsberg AM, Brønstad G (2012) Peptides’ role in autism with emphasis on exorphins. Microb Ecol Health Dis 23.

99. Boukthir S, Matoussi N, Belhadj A, Mammou S, Dlala SB, et al. (2010) [Abnormal intestinal permeability in children with autism]. Tunis Med 88: 685-686.

100. Emanuele E, Orsi P, Boso M, Broglia D, Brondino N, et al. (2010) Lowgrade endotoxemia in patients with severe autism. Neurosci Lett 471: $162-165$.

101. Cade R, Privette R, Fregly M, Rowland N, Sun Z, et al. (2000) Autism and schizophrenia: intestinal disorders. Nutr Neurosci 3: 57-72.

102. Cass H, Gringras P, March J, McKendrick I, O’Hare AE, et al. (2008) Absence of urinary opioid peptides in children with autism. Arch Dis Child 93: 745-750.

103. Shattock P, Hooper M, Waring R (2004) Opioid peptides and dipeptidyl peptidase in autism. Dev Med Child Neurol 46: 357.
104. Ratajczak HV (2011) Theoretical aspects of autism: causes-a review. J Immunotoxicol 8: 68-79.

105. Mayer EA, Padua D, Tillisch K (2014) Altered brain-gut axis in autism: comorbidity or causative mechanisms? Bioessays 36: 933-939.

106. White JF (2003) Intestinal pathophysiology in autism. Exp Biol Med (Maywood) 228: 639-649.

107. Hewitson L (2013) Scientific challenges in developing biological markers for autism. OA Autism 1: 7 .

108. Gilger MA, Redel CA (2009) Autism and the gut. Pediatrics 124: 796798.

109. Erickson CA, Stigler KA, Corkins MR, Posey DJ, Fitzgerald JF, et al. (2005) Gastrointestinal factors in autistic disorder: a critical review. J Autism Dev Disord 35: 713-727. 